\title{
Dual growth factor delivery from bilayered, biodegradable hydrogel composites for spatially-guided osteochondral tissue repair
}

\author{
Steven Lu ${ }^{\mathrm{a}}$, Johnny Lam ${ }^{\mathrm{a}}$, Jordan E. Trachtenberg ${ }^{\mathrm{a}}$, Esther J. Lee ${ }^{\mathrm{a}}$, Hajar Seyednejad ${ }^{\mathrm{a}}$, \\ Jeroen J. J. P. van den Beucken ${ }^{d}$, Yasuhiko Tabata ${ }^{b}$, Mark E. Wong ${ }^{c}$, John A. Jansen ${ }^{d}$,

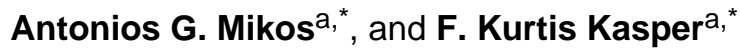

aDepartment of Bioengineering, Rice University, Houston, USA bepartment of Biomaterials, Institute for Frontier Medical Sciences, Kyoto University, Kyoto, Japan 'Department of Surgery, Division of Oral and Maxilofacial Surgery, The University of Texas School of Dentistry at Houston, Houston, USA dDepartment of Biomaterials, Radboud University, Nijmegen Medical Center, Nijmegen, The Netherlands

\begin{abstract}
The present work investigated the use of biodegradable hydrogel composite scaffolds, based on the macromer oligo(poly(ethylene glycol) fumarate) (OPF), to deliver growth factors for the repair of osteochondral tissue in a rabbit model. In particular, bilayered OPF composites were used to mimic the structural layers of the osteochondral unit, and insulin-like growth factor-1 (IGF-1) and bone morphogenetic protein-2 (BMP-2) were loaded into gelatin microparticles and embedded within the OPF hydrogel matrix in a spatially controlled manner. Three different scaffold formations were implanted in a medial femoral condyle osteochondral defect: 1) IGF-1 in the chondral layer, 2) BMP-2 in the subchondral layer, and 3) IGF-1 and BMP-2 in their respective separate layers. The quantity and quality of osteochondral repair was evaluated at 6 and 12 weeks with histological scoring and micro-computed tomography (micro-CT). While histological scoring results at 6 weeks showed no differences between experimental groups, micro-CT analysis revealed that the delivery of BMP-2 alone increased the number of bony trabecular islets formed, an indication of early bone formation, over that of IGF-1 delivery alone. At 12 weeks postimplantation, minimal differences were detected between the three groups for cartilage repair. However, the dual delivery of IGF-1 and BMP-2 had a higher proportion of subchondral bone repair, greater bone growth at the defect margins, and lower bone specific surface than the single delivery of IGF-1. These results suggest that the delivery of BMP-2 enhances subchondral bone
\end{abstract}

\footnotetext{
(C) 2014 Elsevier Ltd. All rights reserved.

"Corresponding Authors: Antonios G. Mikos, Ph.D., Louis Calder Professor, Department of Bioengineering, Rice University, P.O. Box 1892, MS-142, Houston, Texas, 77005-1892, w: 713-348-5355, f: 713-348-4244, mikos@ rice.edu. F. Kurtis Kasper, Ph.D., Senior Faculty Fellow, Department of Bioengineering, Rice University, P.O. Box 1892, MS-142, Houston, Texas, 77005-1892, w: 713-348-3027, f: 713-348-4244, kasper@ rice.edu.

Publisher's Disclaimer: This is a PDF file of an unedited manuscript that has been accepted for publication. As a service to our customers we are providing this early version of the manuscript. The manuscript will undergo copyediting, typesetting, and review of the resulting proof before it is published in its final citable form. Please note that during the production process errors may be discovered which could affect the content, and all legal disclaimers that apply to the journal pertain.
} 
formation and that, while the dual delivery of IGF-1 and BMP-2 in separate layers does not improve cartilage repair under the conditions studied, they may synergistically enhance the degree of subchondral bone formation. Overall, bilayered OPF hydrogel composites demonstrate potential as spatially-guided, multiple growth factor release vehicles for osteochondral tissue repair.

\section{Keywords}

Insulin-like growth factor-1; bone morphogenetic protein-2; subchondral bone; cartilage repair; rabbit model

\section{Introduction}

The osteochondral unit is a multiphasic tissue comprised of two main tissue types: the articulating cartilage responsible for nearly frictionless movement and shock-absorbance, and the subchondral bone indispensible for underlying mechanical support [1-3]. Within the knee, the health of cartilage tissue has been linked to the maintenance of the natural biomechanics of the subchondral bone, and given the contact between cartilage and bone, total joint homeostasis will ultimately rely on the functional restoration of both tissues [4-7]. As a result, tissue engineering strategies to regenerate the osteochondral unit have evolved over the years from monolithic structures to gradient/bilayered scaffolds as a means of controlling treatment to repair the cartilage-bone interface or to target each tissue separately [8-10]. In addition to selecting an appropriate scaffold design, successful regeneration of osteochondral tissue is greatly influenced by the microenvironment in which cells and tissues grow [11]. A number of soluble cues have been implicated in osteochondral wound healing environment [10], and the goal of eliciting the desired phenotypic response from codelivered and/or host progenitor cells in vivo remains a difficult challenge. Due to the numerous signaling pathways involved in osteochondral repair, the delivery of multiple growth factors to improve therapeutic potency is an area of great interest, particularly when both cartilage and bone tissues are considered [12].

The present work seeks to repair osteochondral tissue through the dual delivery of growth factors from acellular, bilayered hydrogel composites. These hydrogel composites are fabricated from a synthetic macromer, oligo(poly(ethylene glycol) fumarate) (OPF), which is synthesized by the esterification of poly(ethylene glycol) (PEG) and fumaryl chloride. The resulting macromer can be crosslinked via the double bonds of the fumarate group to form a hydrogel as well as degrade through hydrolysis of the ester groups [13, 14]. Gelatin microparticles (GMPs) are also embedded within the crosslinked hydrogel matrix to act as both an enzymatically digestible porogen and a delivery vehicle for growth factors [15-17]. These OPF-based hydrogel composites have been used in a number of in vitro and in vivo studies as growth factor delivery vehicles, and allows for comparisons between growth factor types and delivery methods for cartilage and osteochondral tissue repair applications [15-20]. In an effort to mimic the osteochondral unit, these hydrogels are laminated to form two distinct layers as a means of tailoring scaffold composition and drug loading to the cartilage and bone areas of the defect $[18,21,22]$. With this design, the current study incorporates a chondroinductive factor and an osteoinductive factor into the bilayered 
hydrogels in separate layers to facilitate simultaneous cartilage and subchondral tissue repair within an osteochondral defect.

The strategy of spatially incorporated biologics for simultaneous in vivo cartilage and bone repair is a growing area of research: Chen et al. delivered plasmids encoding for transforming growth factor- $\beta 1$ (TGF- $\beta 1$ ) and bone morphogenetic protein-2 (BMP-2) in separate layers of a chitosan-gelatin scaffold [23], Re'em et al. spatially presented TGF- $\beta 1$ and BMP-4 in an alginate-sulfate hydrogel [24], and Mohan et al. and Dormer et al. created sintered poly(D,L-lactic-co-glycolic acid) microsphere scaffolds with reverse gradients of TGF- $\beta 1$ and BMP-2 $[25,26]$. However, an optimal combination of chondrogenic and osteogenic growth factors has yet to be achieved for osteochondral regeneration.

As a result, the main hypothesis of this study is that insulin-like growth factor-1 (IGF-1) delivered from the chondral layer and BMP-2 delivered from the subchondral layer of bilayered OPF hydrogel composites can act synergistically to promote osteochondral tissue repair over the delivery of either growth factor alone. Additionally, it is hypothesized that earlier subchondral bone repair, stimulated by the release of BMP-2, will affect the degree of cartilage repair. IGF-1 is the main anabolic growth factor in articular cartilage and plays a key role in homeostasis by balancing synthesis and break down of proteoglycans by chondrocytes [27]. Additionally, previous studies have demonstrated similar or improved cartilage repair with the single delivery of IGF-1 over the dual delivery of IGF-1 with TGF$\beta 1$ or TGF- $\beta 3$ from bilayered OPF composites in a medial femoral condyle osteochondral defect model [19, 20]. BMP-2 is a potent osteogenic growth factor for inducing de novo bone formation in ectopic and orthotopic sites and has been shown to improve subchondral bone formation when delivered to osteochondral defects [28-34]. Additionally, BMP-2 is expressed throughout the entire chondrogenic process of mesenchymal stem cells (MSCs), from condensation to proliferation, differentiation, maturation, and calcification [10]. With the ability to induce the growth of both cartilage and bone tissue, several research groups have delivered BMP-2 from monolithic scaffolds to osteochondral defects and have improved cartilage repair over blank scaffolds [32, 34, 35]. However, the current study isolates the incorporation of BMP-2 to the subchondral layer to maximize its osteoinductive potential and reduce its direct morphogenetic effects on progenitor cell chondrogenesis in the chondral layer.

To test the two hypotheses aforementioned, the current study 1) investigates the repair of cartilage and subchondral bone tissue in an osteochondral defect facilitated by bilayered hydrogel composites delivering IGF-1 and/or BMP-2 incorporated in a spatially controlled manner, 2) evaluates the degree of subchondral bone repair with micro-computed tomography (micro-CT) and osteochondral tissue repair through histological scoring, and 3) compares the extent of cartilage and subchondral bone repair at 6 week and 12 week time points. 


\section{Materials and Methods}

\subsection{Oligo(poly(ethylene glycol) fumarate) synthesis}

OPF was synthesized from PEG (Sigma-Aldrich, St. Louis, MO) with a nominal molecular weight (MW) of 35,000 according to methods previously developed in our laboratory [13, 14]. Gel permeation chromatography was used to characterize the OPF macromer to give a number average molecular weight of 50,400 $\pm 900 \mathrm{Da}$ and a weight average molecular weight of $127,400 \pm 3,200 \mathrm{Da}$. Prior to the fabrication of hydrogel implants, OPF was sterilized for 12 hours by exposure to ethylene oxide (EO) according to established protocols $[20,36]$.

\subsection{Gelatin microparticle fabrication and growth factor loading}

Using established methods, GMPs were fabricated using acidic gelatin (Nitta Gelatin, Osaka, Japan) with an isoelectric point (IEP) of 5.0 and crosslinked in $40 \mathrm{mM}$ glutaraldehyde (Sigma-Aldrich, St. Louis, MO), followed by glycine quenching of unreacted glutaraldehyde [16, 37]. Following lyophilization, GMPs of 50-100 $\mu \mathrm{m}$ in diameter were obtained through sieving and EO sterilized for 12 hours prior to GF loading. GMPs were then partially swelled with either phosphate buffered saline (PBS) or a GF solution at a ratio of $5 \mu \mathrm{L}$ solution to $1 \mathrm{mg}$ dried GMP for $15 \mathrm{hrs}$ at $4^{\circ} \mathrm{C}$ before hydrogel encapsulation according to previously described methods [15, 17]. In particular, $225 \mu \mathrm{L}$ of PBS, IGF-1 solution, or BMP-2 solution was combined with $45 \mathrm{mg}$ of GMPs. Recombinant human IGF-1 (Peprotech, Rocky Hill, NJ) was reconstituted with PBS to a GF loading concentration of $7.95 \mu \mathrm{g}$ per mL solution and loaded onto GMPs to give a total of $5.6 \mathrm{ng}$ IGF-1 per hydrogel construct. Recombinant human BMP-2 (Peprotech, Rocky Hill, NJ) was reconstituted with a mixture of $\mathrm{ddH}_{2} \mathrm{O}$ and PBS to a GF loading concentration of $712 \mu \mathrm{g}$ per $\mathrm{mL}$ solution to give a total of $1 \mu \mathrm{g}$ BMP-2 per hydrogel construct.

\subsection{Bilayered hydrogel composite fabrication}

Bilayered hydrogel composites were fabricated via a two-step crosslinking procedure similar to methods previously described $[19,20]$. Using a $2 \mathrm{~mm} \times 2 \mathrm{~mm}$ cylindrical teflon mold, the subchondral layer was first partially crosslinked, then the chondral layer was crosslinked on top of the subchondral layer. Specifically, to prepare the OPF precursor solution, $113 \mathrm{mg}$ of OPF and $37 \mathrm{mg}$ of PEGDA (3,400 Da MW, Laysan Bio, Arab, AL) were dissolved in 353 $\mu \mathrm{L}$ of PBS and set at room temperature for 45 minutes to eliminate air bubbles. $46.8 \mu \mathrm{L}$ each of the thermal radical initiators, $0.3 \mathrm{M}$ ammonium persulfate (APS, Sigma-Aldrich, St. Louis, MO) and 0.3 M N,N, $\mathrm{N}^{\prime}, \mathrm{N}^{\prime}$-tetramethylethylenediamine (TEMED, Sigma-Aldrich, St. Louis, MO), were then mixed into the polymer solution. The addition of APS and TEMED at the concentrations used are expected to have minimal toxic effects on the surrounding tissue post-implantation, particularly in consideration of previous studies showing high viability of encapsulated cells in OPF-based hydrogels following the fabrication process [22, 38, 39]. Lastly, $45 \mathrm{mg}$ of GMPs swollen with either $225 \mu \mathrm{L}$ of PBS or a GF solution were added to the solution and mixed carefully to avoid the formation of bubbles. The polymerGMP mixture was then injected into the bottom two-thirds of the teflon mold for the subchondral layer and partially crosslinked at $37^{\circ} \mathrm{C}$ for 5 minutes. A similar polymer-GMP mixture was then prepared for the chondral layer and injected on the subchondral layer and 
crosslinked at $37^{\circ} \mathrm{C}$ for 25 minutes. Table 1 highlights the three groups used in the study with BMP-2 delivered from the subchondral layer and IGF-1 delivered from the chondral layer of the bilayered hydrogel composites. Prior to implantation in an osteochondral defect, hydrogels were transferred with a drop of sterile PBS to prevent the hydrogel composites from drying. Bilayered hydrogel composites at equilibrium swelling were cylinders $3 \mathrm{~mm} \times$ $3 \mathrm{~mm}$ in size (data not shown).

\subsection{Animal surgery}

A total of 36 male, skeletally mature (i.e., 6 months old) New Zealand White rabbits were used as determined by a power analysis and in consideration of previous studies [18-20,36]. Each animal received bilateral implants (one per knee) and a total of 10-12 hydrogels were implanted in 5-6 rabbits per group per time point. It should be noted that one rabbit for the 6 week BMP-2 group had complications on the surgery table due to previously undetected Pasteurella infection and was excluded from the study. All surgical procedures and subsequent animal care were approved by the Institutional Animal Care and Use Committees of Rice University and The University of Texas Health Science Center at Houston. Briefly, anesthesia was induced prior to surgery by subcutaneous injections of ketamine $(25-40 \mathrm{mg} / \mathrm{kg})$ and acepromazine $(1-2 \mathrm{mg} / \mathrm{kg})$. General anesthesia was maintained by inhalation of isoflurane (2-3\%) and oxygen. Rabbits were immobilized on their backs and the hair from both hind legs was shaven. The medial femoral condyle was then exposed through a medial parapatellar longitudinal incision, incision of synovial capsule, and lateral luxation of the patella. An osteochondral defect ( $3 \mathrm{~mm}$ in diameter and $3 \mathrm{~mm}$ in depth) was then created through serial drilling $(2 \mathrm{~mm} \rightarrow 2.75 \mathrm{~mm} \rightarrow 3 \mathrm{~mm})$ with a dental drill under irrigation (Osseo Scientific, Toms River, NJ). The defect was then flushed with saline, dried with gauze, and the hydrogel composite was implanted to allow for swelling to the same dimensions of the defect. This procedure was then repeated for the contralateral knee using a hydrogel of the same formulation. The synovial capsule and skin on both knees were then closed with 3-0 and 4-0 Vicryl sutures, respectively. To minimize post-operative discomfort, Carprofen $(4 \mathrm{mg} / \mathrm{kg}$ ) was administered subcutaneously for two days postoperatively.

\subsection{Tissue processing and histological scoring}

Rabbits were euthanized at 6 or 12 weeks post-surgery with an intravenous injection of Beuthanasia $(0.22 \mathrm{~mL} / \mathrm{kg})$. The tissue surrounding the medial femoral condyle defect was retrieved en bloc, cut with a low-speed diamond saw to isolate the medial femoral condyle, fixed in 10\% neutral buffered formalin for no more than 72 hours, decalcified in EDTA solution for 6 weeks, dehydrated through a graded series of ethanol baths, and embedded in paraffin. Longitudinal sections of $5 \mu \mathrm{m}$ thickness were taken from the center (within the central $1 \mathrm{~mm}$ ) and the peripheral edges (within the medial and lateral $1 \mathrm{~mm}$ ) with a microtome (Leica RM2165). Sections from each location were stained with hematoxylin and eosin (H\&E), Safranin O/Fast Green (Saf.O), and van Gieson's Picrofuchsin (VGP). Histological sections were blindly and independently scored by three evaluators (J.L., J.T., F.K.K.) using a previously established histological scoring system for osteochondral defects as shown in Table $2[19,20,36]$. H\&E and VGP sections were primarily used for overall defect and subchondral bone evaluation parameters while Saf.O sections were primarily 
used for cartilage evaluation parameters. Independent of the histological scoring system, the presence of complete subchondral bone plate bridging within the defect and neo-surface depressions below the tidemark were also evaluated for each histological section. It should be noted that sections from one sample in the 12 week BMP-2 group had large artifacts that resulted in the inability to accurately score the histological images and were excluded in the histological analysis.

\subsection{Micro-computed tomography}

All femoral condyles were scanned using a SkyScan 1172 micro-CT imaging system (Bruker, Kontich, Belgium) prior to EDTA decalcification. A voltage of $100 \mathrm{kV}$ and a current of $100 \mu \mathrm{A}$ with a nominal resolution of $10 \mu \mathrm{m}$ pixel $^{-1}$ was used. Image data were reconstructed using NRecon v1.6 and analyzed with CTAn v1.10, where thresholding levels of gray values (70-255) were set for all samples. A circular region of interest $3 \mathrm{~mm}$ in diameter was selected for a top-down analysis and transverse images were used to position the upper boundary of the volume of interest (VOI) at the surface of the cartilage layer of the cylindrical defect. Due to the presence of two distinct bone morphologies in the subchondral tissue, the bone plate and trabecular bone, two VOIs were analyzed separately: the upper .85 $\mathrm{mm}$ for the cartilage and cortical region $(\mathrm{C} \& \mathrm{C})$ and the lower $2.15 \mathrm{~mm}$ for the trabecular region. Due to the potential for the subchondral bone plate to migrate upward during osteochondral repair, the cartilage region was also included for the C\&C VOI [40]. Parameters analyzed for the $\mathrm{C} \& \mathrm{C}$ and trabecular VOI include bone mineral density (BMD), percent bone volume (BV/TV), intersection surface (i.S.), bone specific surface (BS/BV), and object number (Obj.N.). Table 3 further explains each of these parameters. Independent of the micro-CT parameter analysis, the presence of complete subchondral bone plate filling and bridging was also evaluated for each sample.

\subsection{Statistical analysis}

A significance level of $\mathrm{p}<0.05$ was used for all statistical analysis through JMP Pro v10.0.2. For histological scoring analysis, a one-way analysis was performed to analyze the potential effect of experimental group, time point, location within the defect, and knee joint. The Kruskal-Wallis test of variance was used and post-hoc analysis done with the SteelDwass All Pairs test. For micro-CT analysis, a one-way analysis was performed to analyze the potential effects of experimental group and time point. A one-way ANOVA was used to test variance and Tukey HSD All Pairs test was used to compare means.

\section{Results}

\subsection{Histological Observations and Scoring}

Sections from three locations (lateral edge, medial edge, and center) were obtained per sample for histological evaluation. Representative images for all groups at week 6 and week 12 are shown in Figures 1 and 2, respectively. Figure 3 displays the mean histological scores from the 11 parameters examined as described in Table 2, and the distribution of scores for bone morphology, cartilage morphology, and cartilage thickness are shown in Figure 4. 
Following histological scoring of all images, the potential effects of experimental group, time point (week 6 vs. week 12), location within the defect (edges vs. center), and knee joint (right vs. left) were evaluated. Location within the defect and knee joint did not have any statistical impact on the 11 histological parameters scored, indicating that there was no major intra-defect variability nor bilateral implant variability. However, it should be noted that neo-tissue formation was seen to develop most commonly from the defect margins in the subchondral and chondral regions. The effect of time point for all samples for the histological parameters scored is shown in Table 4.

3.1.1 Overall Evaluation-In the overall evaluation of the defect, the histological scores for overall tissue filling generally mirrored those of overall implant degradation (Figure 3a). At week 6, there were no differences between groups in overall filling and degradation and none of the samples were seen to have complete degradation of the hydrogel composite implant as seen by representative images in Figure 1. Additionally, the remaining hydrogel was located in the subchondral region of the osteochondral defect for all samples. At week 12 , greater degradation of the implants was seen with $44 \%, 63 \%$, and $41 \%$ of histological images showing no presence of remaining hydrogel composite for IGF-1, BMP-2, and Both groups, respectively. More specifically, when all three sections from one sample had no presence of the remaining implant, 3/12 IGF-1 samples, 4/11 BMP-2 samples, and 0/12 Both samples showed complete degradation. When all samples at week 12 were compared against week 6 samples, there was a significant effect of time point, with week 12 samples having greater scores for overall filling and degradation than week 6 samples.

3.1.2 Subchondral Evaluation-Similar to overall evaluation, evaluation of the subchondral bone revealed no differences between experimental groups at 6 weeks. In the majority of the samples, the implant was surrounded by a fibrous capsule (Figure 1F), giving the histological image a score of 1 for bone morphology. Hypertrophic and calcified cartilage were also seen in many of the sections (Figure 1G-I), indicative of cartilage resorption and bone formation processes. At 12 weeks, while there were no differences for bone filling between groups, the BMP-2 group had better bone morphology scores compared to the Both group as seen in Figure 3b. The BMP-2 group also had the greatest percentage of sections with trabecular bone at $36 \%$, whereas $17 \%$ and $12 \%$ of sections for IGF-1 and Both, respectively, showed trabecular bone for the entire subchondral region (Figure $4 \mathrm{a}$ ).

However, the majority of all samples at both week 6 and 12 had cortical and/or trabecular bone formation from the subchondral defect margins and all received a score of 3 for new tissue bonding with the adjacent bone (Figure $3 \mathrm{~b}$ ). The repair of the subchondral bone plate was also assessed, with $22 \%, 22 \%$, and $50 \%$ of histological sections for IGF-1, BMP-2, and Both groups at week 12, respectively, showing repaired bone plate tissue in line with the adjacent bone plate. More specifically, 0/12, 2/11, and 3/12 samples for IGF-1, BMP-2, and Both groups respectively showed complete bone plate bridging with all three sections per samples having presence of a continuous bone plate. No repair of the bone plate was seen in any of the 6 week samples. In a comparison between 6 week and 12 week samples for subchondral bone histological scores, 12 week samples had statistically greater bone filling and bone morphology scores than 6 week samples. 
3.1.3 Cartilage Evaluation-Cartilage repair was evaluated with the parameters of cartilage morphology, cartilage thickness, surface regularity, chondrocyte clustering, neocartilage glycosaminoglycan (GAG) and cell content, and adjacent GAG and cell content (Figure 3c). For neo-cartilage morphology, there was no difference between time points nor between groups. However, the 12 week Both group had the greatest percentage of samples with articular cartilage repair while many 12 week BMP-2 samples had fibrocartilage, and the 12 week IGF-1 group had the most sections with fibrous cartilage formation (Figure 4b). In having the most sections with articular cartilage, the 12 week Both group also had the best scores for chondrocyte clustering and a significant difference from the 12 week BMP-2 group (Figure 3c). Although harvested at an earlier time point, many of the 6 week samples had hyaline cartilage formation with intense GAG staining. However, these samples also tended to have hypercellularity in the neo-cartilage tissue with cell clustering or unorganized cells (Figure 1G-I). As a result, 6 week samples overall had statistically greater neocartilage GAG and cellularity scores than 12 week samples.

The thickness of neo-cartilage was also greater at 6 weeks compared to 12 weeks with approximately $80 \%$ of all 6 week samples having similar or thicker neo-cartilage tissue with respect to surrounding cartilage (Figure $4 \mathrm{c}$ ). In contrast, approximately $70 \%$ of all 12 week samples had similar or thinner neo-cartilage tissue. In examining surface regularity, there were no differences among all groups. However, the nature of the surface regularity differed between 6 and 12 week samples. 12 week samples generally exhibited more irregularities on the repaired tissue surface (e.g., small fissures, bumps, etc.), while 6 week samples had more depressions in the cartilage compared to the surrounding tissue (Figure 1). 30\%, 21\%, and $34 \%$ of IGF-1, BMP-2, and Both sections, respectively, at 6 weeks had the surface of the neo-tissue below that of the surrounding tidemark. On the other hand, $8 \%, 16 \%$, and $21 \%$ of IGF-1, BMP-2, and Both sections, respectively, at 12 weeks had surface depressions that dipped below the adjacent cartilage. In evaluating the GAG and cell content of the adjacent cartilage, normal GAG staining and cellularity was seen in most 12 week samples, with some sections demonstrating normal GAG staining on one side of the defect, and reduced staining on the other side. This was more apparent for 6 week samples as seen in Figure 1G. As a result, 12 week samples overall had statistically greater scores for adjacent GAG and cell content compared to their 6 week counterparts.

\subsection{Micro-Computed Tomography Analysis}

The degree of subchondral bone repair was measured through quantitative analysis of two different VOIs (Figure 5a), C\&C and trabecular, for five morphometric bone parameters: BMD, BV/TV, i.S., BS/BV, and Obj.N. (Figure 6). The effects of time point for all samples was also analyzed (Table 4). No differences were seen among the three groups at week 6 for BMD, BV/TV and i.S. However, group BMP-2 had greater BS/BV in the C\&C region and a higher Obj.N. in both VOIs than group IGF-1 at week 6 (Figure 6d-e). Figure 5b-c show representative images of the incomplete subchondral tissue repair for a 6 week sample (6 week Both). By 12 weeks, micro-CT analysis showed that the delivery of both growth factors resulted in greater BMD, BV/TV, and i.S. compared to that of the IGF-1 only group for the $\mathrm{C} \& \mathrm{C}$ region. Additionally, the 12 week Both group had greater i.S. and lower BS/BV than the 12 week IGF-1 group for the trabecular VOI. Comparison between 6 week and 12 
week samples revealed that 12 week samples had a higher BMD, BV/TV ratio, and greater i.S. area than the 6 week samples, and conversely, 6 week samples had greater BS/BV and Obj.N. than 12 week samples in both $\mathrm{C} \& \mathrm{C}$ and trabecular regions. The repair of the subchondral bone plate was also assessed through observation of the micro-CT images. In accordance with the histological images, no bone plate was fully repaired for the 6 week samples and 0,2, and 3 samples at 12 weeks for the IGF-1, BMP-2, and Both groups respectively showed complete bridging and filling of the defect area with compact bone. However, the regeneration of the bone plate was not complete as seen in Figure 5d, with the repair bone in the defect showing a marked difference in mineral density compared to the adjacent compact bone.

\section{Discussion}

The primary objective of this study was to determine the effect of dual growth factor release using IGF-1 from the chondral layer and BMP-2 from the subchondral layer of bilayered OPF hydrogel composites on osteochondral tissue repair. The degree of osteochondral repair was evaluated at 6 and 12 weeks and histological scoring and micro-CT analyses were used. Specifically, we investigated 1) if early subchondral bone repair, as measured at 6 weeks, would result in better bone and cartilage repair at 12 weeks and 2) whether the dual delivery of IGF-1 and BMP-2 in separate layers would act synergistically to improve osteochondral tissue regeneration over the delivery of either growth factor alone.

As a potent morphogenetic factor, BMP-2 has been delivered for a number of ectopic and orthotopic bone regeneration applications and has been used to stimulate chondrogenesis of MSCs in vitro as well as improve both cartilage and bone repair in osteochondral defects in vivo [25, 26, 29, 41-43]. Since it can directly affect osteogenesis and chondrogenesis of MSCs, this study loaded BMP-2 onto GMPs that were localized in the subchondral layer of bilayered scaffolds in order to focus the morphogenetic potential of BMP-2 on bone repair. However, we acknowledge that BMP-2 may diffuse into chondral space, or conversely, IGF-1 may diffuse into the subchondral space over the course of $6-12$ weeks. Further research tracking the diffusion of growth factors or confining the delivery of growth factors to their respective regions could help address this limitation. Even so, previous studies studying subchondral bone repair in a trochlear groove defect show that upward migration of the subchondral bone plate and thinning of cartilage is the natural course of osteochondral repair $[40,44]$, and that the presence of BMP-2 in the chondral region of the defect does not exacerbate this process $[32,34]$. In this study, only two samples at week 12 , one in the BMP-2 group and the other in the Both group, had repair of the subchondral bone extending beyond the tidemark of the adjacent tissue but below the joint surface.

In order to examine early subchondral bone repair, we evaluated the defects at 6 weeks with both histological scoring and micro-CT analysis. We saw that there were no differences in the quantity of bone formation at 6 weeks, as evaluated by bone filling, BMD, and BV/TV. This was most likely due to the incomplete degradation of the implants, which was present in all 6 week samples. Bone morphology scoring, bond bonding scoring, and i.S. measurements also showed no differences between the experimental groups. However, micro-CT analysis revealed a difference with an increase in BS/BV in the C\&C VOI and a 
higher Obj.N. count for group BMP-2 in both VOIs over group IGF-1 at 6 weeks. This could signify an increase in the number of woven bone islets in the subchondral space, which is a commonly observed phenomenon in early bone repair for segmental defects [29, 41, 45]. Other studies delivering BMP-2 to osteochondral defects have also demonstrated accelerated subchondral bone formation with the delivery of BMP-2 [32, 34]. In particular, Sellers et al. found that when BMP-2 was delivered in a collagen sponge to a trochlear groove defect, most of the new-bone formation took place between two and four weeks, and new subchondral bone tended to be more sclerotic between four and eight weeks [32]. Thus, the single delivery of BMP-2 may have enhanced subchondral ossification over that of the single delivery of IGF-1, resulting in a greater number of isolated bony trabeculae at the 6 week time point.

Histological and micro-CT analysis revealed greater differences in subchondral bone repair at 12 weeks. With histological scoring, the delivery of BMP-2 alone resulted in better scores for bone morphology than the dual delivery of IGF-1 and BMP-2. This could potentially be due to the increased percentage of samples with complete implant degradation in the BMP-2 only group (4/11 samples) compared to the Both group (0/12 samples), resulting in more sections with trabecular bone and no fibrous tissue present. With micro-CT, in-depth scrutiny of the repair bone was provided in both the trabecular and cortical regions. Analysis of BMD, BV/TV, i.S., and BS/BV revealed that the delivery of both BMP-2 and IGF-1 resulted in better subchondral bone repair than IGF-1 alone. Additionally, group Both at 12 weeks had the highest bone mineral density, percent bone volume, and bone intersection surface in the cortical region than all other groups, regardless of the time point.

Compounded with the fact that the dual delivery group had the most sections with complete bone plate bridging with the adjacent bone plate at 50\% suggests that the delivery of IGF-1 and BMP-2 synergistically improved subchondral bone plate repair over either growth factor alone. Indeed, the dual delivery of IGF-1 and BMP-2 has been shown to enhance alkaline phosphatase expression and calcium deposition from periodontal ligament fibroblasts over either growth factor alone [46]. The combination of both growth factors has also been shown to improve bone formation in a rabbit femoral osteotomy defect over BMP-2 alone at 4 and 8 week time points [47].

When the extent of cartilage repair at 12 weeks was examined through histological scoring, the dual delivery of IGF-1 and BMP-2 resulting in less chondrocyte clustering than the single delivery of BMP-2. While group Both had the highest percentage of sections with articular cartilage and well-organized chondrocytes in a columnar fashion, there were no statistical differences with the other groups. Interestingly, the delivery of IGF-1 alone resulted in better adjacent GAG and cell content at 12 weeks than all 6 week groups. A similar result was achieved in previous studies where the delivery of IGF-1 alone also gave the highest scores for adjacent GAG and cell content $[19,20]$. This may highlight the importance of IGF-1 in regulating the homeostasis of native cartilage tissue and balancing proteoglycan synthesis and breakdown.

Overall, with minimal differences in cartilage repair at 12 weeks it is difficult to identify a correlation between early bone repair and improved cartilage repair at 12 weeks. Additionally, the delivery of IGF-1 together with BMP-2 from bilayered OPF hydrogel 
composites did not improve cartilage repair over either growth factor alone. This could be due to the different layers from which IGF-1 and BMP-2 were delivered. Previous in vitro research has demonstrated the synergistic effects of BMP-2 and IGF-1 on chondrocytes seeded in agarose gels in increasing aggregate modulus and GAG production over either growth factor alone [42]. However, in a porous silk scaffold with reverse gradients of BMP-2 and IGF-1, seeded human MSCs exhibited chondrogenic and osteogenic differentiation along the BMP-2 gradient, with the greatest changes in human MSC differentiation occurring in the region with the highest BMP-2 concentration [48]. In contrast, cells in the region with the highest IGF-1 and lowest BMP-2 concentration retained MSC morphology (spindle-shaped). With this in mind, the improved subchondral bone repair with IGF-1 and BMP-2 and the lack of statistical differences in cartilage repair between experimental groups suggests that the BMP-2 delivered from the subchondral layer of bilayered OPF composites had a greater direct effect on bone repair than on cartilage repair.

\section{Conclusion}

IGF-1 and BMP-2 were loaded into GMPs in the chondral layer and subchondral layer, respectively, of bilayered OPF hydrogel composites either separately or together. These three formulations were tested for osteochondral repair in a rabbit model at 6 and 12 weeks. While histological scoring showed no differences in cartilage and bone repair at 6 weeks, micro-CT revealed that the single delivery of BMP-2 increased bone specific surface and the number of bony trabecular islets over the single delivery of IGF-1. At 12 weeks, micro-CT also showed an increase in bone mineral density, percent bone volume, and intersection surface, and a decrease in bone specific surface for the dual delivery of IGF-1 and BMP-2 over the single delivery of IGF-1. However, there was no significant difference between the three groups on cartilage morphology at 12 weeks. These results demonstrate that the delivery of BMP-2 can accelerate bone formation at an early time point and that the delivery of both growth factors in separate layers has a synergistic effect on subchondral bone repair, but not on cartilage repair, in an osteochondral defect. Finally, the outcomes of this study can help inspire future spatially-controlled, multiple growth factor delivery strategies for osteochondral tissue regeneration.

\section{Acknowledgments}

This work was supported by the National Institutes of Health (R01-AR048756). The authors acknowledge the technical assistance of Ms. Natasja van Dijk with the histological preparation of specimens and Mr. Brian C. Dawson with micro-CT evaluation. The authors would also like to thank the registered veterinary technicians at The University of Texas Health Science Center for their support during the animal procedures.

\section{References}

1. Vinatier C, Mrugala D, Jorgensen C, Guicheux J, Noel D. Cartilage engineering: A crucial combination of cells, biomaterials and biofactors. Trends Biotechnol. 2009; 27:307-14. [PubMed: 19329205]

2. Vinatier C, Bouffi C, Merceron C, Gordeladze J, Brondello JM, Jorgensen C, et al. Cartilage tissue engineering: Towards a biomaterial-assisted mesenchymal stem cell therapy. Curr Stem Cell Res Ther. 2009; 4:318-29. [PubMed: 19804369] 
3. Danisovic L, Varga I, Polak S. Growth factors and chondrogenic differentiation of mesenchymal stem cells. Tissue Cell. 2012; 44:69-73. [PubMed: 22185680]

4. Radin EL, Ehrlich MG, Chernack R, Abernethy P, Paul IL, Rose RM. Effect of repetitive impulsive loading on knee joints of rabbits. Clin Orthop Relat Res. 1978:288-93. [PubMed: 657637]

5. Serink MT, Nachemson A, Hansson G. Effect of impact loading on rabbit knee joints. Acta Orthop Scand. 1977; 48:250-62. [PubMed: 920116]

6. Radin EL, Rose RM. Role of subchondral bone in the initiation and progression of cartilage damage. Clin Orthop Relat Res. 1986:34-40. [PubMed: 3780104]

7. Goldring SR. Cross-talk between subchondral bone and articular cartilage in osteoarthritis. Arthrit Res Ther. 2012:14.

8. Keeney M, Pandit A. The osteochondral junction and its repair via bi-phasic tissue engineering scaffolds. Tissue Eng Part B Rev. 2009; 15:55-73. [PubMed: 19207035]

9. O'Shea TM, Miao XG. Bilayered scaffolds for osteochondral tissue engineering. Tissue Eng Part B Rev. 2008; 14:447-64. [PubMed: 18844605]

10. Santo VE, Gomes ME, Mano JF, Reis RL. Controlled release strategies for bone, cartilage, and osteochondral engineering-part i: Recapitulation of native tissue healing and variables for the design of delivery systems. Tissue Eng Part B Rev. 2013; 19:308-26. [PubMed: 23268651]

11. Chen FM, Zhang M, Wu ZF. Toward delivery of multiple growth factors in tissue engineering. Biomaterials. 2010; 31:6279-308. [PubMed: 20493521]

12. Santo VE, Gomes ME, Mano JF, Reis RL. Controlled release strategies for bone, cartilage, and osteochondral engineering-part ii: Challenges on the evolution from single to multiple bioactive factor delivery. Tissue Eng Part B Rev. 2013; 19:327-52. [PubMed: 23249320]

13. Jo S, Shin H, Shung AK, Fisher JP, Mikos AG. Synthesis and characterization of oligo(poly(ethylene glycol) fumarate) macromer. Macromolecules. 2001; 34:2839-44.

14. Kinard LA, Kasper FK, Mikos AG. Synthesis of oligo(poly(ethylene glycol) fumarate). Nat Protoc. 2012; 7:1219-27. [PubMed: 22653160]

15. Holland TA, Tabata Y, Mikos AG. In vitro release of transforming growth factor-beta 1 from gelatin microparticles encapsulated in biodegradable, injectable oligo(poly(ethylene glycol) fumarate) hydrogels. J Control Release. 2003; 91:299-313. [PubMed: 12932709]

16. Holland TA, Tabata Y, Mikos AG. Dual growth factor delivery from degradable oligo(poly(ethylene glycol) fumarate) hydrogel scaffolds for cartilage tissue engineering. J Control Release. 2005; 101:111-25. [PubMed: 15588898]

17. Holland TA, Tessmar JKV, Tabata Y, Mikos AG. Transforming growth factor-beta 1 release from oligo(poly(ethylene glycol) fumarate) hydrogels in conditions that model the cartilage wound healing environment. J Control Release. 2004; 94:101-14. [PubMed: 14684275]

18. Holland TA, Bodde EWH, Baggett LS, Tabata Y, Mikos AG, Jansen JA. Osteochondral repair in the rabbit model utilizing bilayered, degradable oligo(poly(ethylene glycol) fumarate) hydrogel scaffolds. J Biomed Mater Res A. 2005; 75A:156-67. [PubMed: 16052490]

19. Holland TA, Bodde EWH, Cuijpers VMJ, Baggett LS, Tabata Y, Mikos AG, et al. Degradable hydrogel scaffolds for in vivo delivery of single and dual growth factors in cartilage repair. Osteoarthritis Cartilage. 2007; 15:187-97. [PubMed: 16965923]

20. Kim K, Lam J, Lu S, Spicer PP, Lueckgen A, Tabata Y, et al. Osteochondral tissue regeneration using a bilayered composite hydrogel with modulating dual growth factor release kinetics in a rabbit model. J Control Release. 2013; 168:166-78. [PubMed: 23541928]

21. Guo X, Park H, Liu GP, Liu W, Cao YL, Tabata Y, et al. In vitro generation of an osteochondral construct using injectable hydrogel composites encapsulating rabbit marrow mesenchymal stem cells. Biomaterials. 2009; 30:2741-52. [PubMed: 19232711]

22. Guo X, Liao J, Park H, Saraf A, Raphael RM, Tabata Y, et al. Effects of tgf-beta 3 and preculture period of osteogenic cells on the chondrogenic differentiation of rabbit marrow mesenchymal stem cells encapsulated in a bilayered hydrogel composite. Acta Biomater. 2010; 6:2920-31. [PubMed: 20197126]

23. Chen JN, Chen HA, Li P, Diao HJ, Zhu SY, Dong L, et al. Simultaneous regeneration of articular cartilage and subchondral bone in vivo using mscs induced by a spatially controlled gene delivery system in bilayered integrated scaffolds. Biomaterials. 2011; 32:4793-805. [PubMed: 21489619] 
24. Re'em T, Witte F, Willbold E, Ruvinov E, Cohen S. Simultaneous regeneration of articular cartilage and subchondral bone induced by spatially presented tgf-beta and bmp- 4 in a bilayer affinity binding system. Acta Biomater. 2012; 8:3283-93. [PubMed: 22617742]

25. Dormer NH, Singh M, Zhao L, Mohan N, Berkland CJ, Detamore MS. Osteochondral interface regeneration of the rabbit knee with macroscopic gradients of bioactive signals. J Biomed Mater Res A. 2012; 100A:162-70. [PubMed: 22009693]

26. Mohan N, Dormer NH, Caldwell KL, Key VH, Berkland CJ, Detamore MS. Continuous gradients of material composition and growth factors for effective regeneration of the osteochondral interface. Tissue Eng Part A. 2011; 17:2845-55. [PubMed: 21815822]

27. Schmidt MB, Chen EH, Lynch SE. A review of the effects of insulin-like growth factor and platelet derived growth factor on in vivo cartilage healing and repair. Osteoarthritis Cartilage. 2006; 14:403-12. [PubMed: 16413799]

28. Vo TN, Kasper FK, Mikos AG. Strategies for controlled delivery of growth factors and cells for bone regeneration. Adv Drug Del Rev. 2012; 64:1292-309.

29. Yamamoto M, Takahashi Y, Tabata Y. Enhanced bone regeneration at a segmental bone defect by controlled release of bone morphogenetic protein-2 from a biodegradable hydrogel. Tissue Eng. 2006; 12:1305-11. [PubMed: 16771643]

30. Yamamoto M, Takahashi Y, Tabata Y. Controlled release by biodegradable hydrogyels enhances the ectopic bone formation of bone morphogenetic protein. Biomaterials. 2003; 24:4375-83. [PubMed: 12922150]

31. Patel ZS, Young S, Tabata Y, Jansen JA, Wong MEK, Mikos AG. Dual delivery of an angiogenic and an osteogenic growth factor for bone regeneration in a critical size defect model. Bone. 2008; 43:931-40. [PubMed: 18675385]

32. Sellers RS, Peluso D, Morris EA. The effect of recombinant human bone morphogenetic protein-2 (rhbmp-2) on the healing of full-thickness defects of articular cartilage. J Bone Joint Surg Am. 1997; 79A:1452-63. [PubMed: 9378731]

33. Lopiz-Morales Y, Abarrategi A, Ramos V, Moreno-Vicente C, Lopez-Duran L, Lopez-Lacomba $\mathrm{JL}$, et al. In vivo comparison of the effects of rhbmp-2 and rhbmp-4 in osteochondral tissue regeneration. Eur Cell Mater. 2010; 20:367-78. [PubMed: 21154243]

34. Tokuhara Y, Wakitani S, Imai Y, Kawaguchi A, Fukunaga K, Kim M, et al. Repair of experimentally induced large osteochondral defects in rabbit knee with various concentrations of escherichia coli-derived recombinant human bone morphogenetic protein-2. Int Orthop. 2010; 34:761-67. [PubMed: 19529936]

35. Yang HS, La WG, Bhang SH, Kim HJ, Im GI, Lee H, et al. Hyaline cartilage regeneration by combined therapy of microfracture and long-term bone morphogenetic protein-2 delivery. Tissue Eng Part A. 2011; 17:1809-18. [PubMed: 21366427]

36. Guo X, Park H, Young S, Kretlow JD, van den Beucken JJ, Baggett LS, et al. Repair of osteochondral defects with biodegradable hydrogel composites encapsulating marrow mesenchymal stem cells in a rabbit model. Acta Biomater. 2010; 6:39-47. [PubMed: 19660580]

37. Tabata Y, Hijikata S, Muniruzzaman M, Ikada Y. Neovascularization effect of biodegradable gelatin microspheres incorporating basic fibroblast growth factor. J Biomater Sci Polym Ed. 1999; 10:79-94. [PubMed: 10091924]

38. Park H, Temenoff JS, Holland TA, Tabata Y, Mikos AG. Delivery of tgf-beta 1 and chondrocytes via injectable, biodegradable hydrogels for cartilage tissue engineering applications. Biomaterials. 2005; 26:7095-103. [PubMed: 16023196]

39. Temenoff JS, Park H, Jabbari E, Conway DE, Sheffield TL, Ambrose CG, et al. Thermally crosslinked oligo(poly(ethylene glycol) fumarate) hydrogels support osteogenic differentiation of encapsulated marrow stromal cells in vitro. Biomacromolecules. 2004; 5:5-10. [PubMed: 14715001]

40. Orth P, Cucchiarini M, Kaul G, Ong MF, Graber S, Kohn DM, et al. Temporal and spatial migration pattern of the subchondral bone plate in a rabbit osteochondral defect model. Osteoarthritis Cartilage. 2012; 20:1161-69. [PubMed: 22771776] 
41. Hollinger JO, Schmitt JM, Buck DC, Shannon R, Joh SP, Zegzula HD, et al. Recombinant human bone morphogenetic protein-2 and collagen for bone regeneration. J Biomed Mater Res. 1998; 43:356-64. [PubMed: 9855194]

42. Elder BD, Athanasiou KA. Systematic assessment of growth factor treatment on biochemical and biomechanical properties of engineered articular cartilage constructs. Osteoarthritis Cartilage. 2009; 17:114-23. [PubMed: 18571441]

43. Puetzer JL, Petitte JN, Loboa EG. Comparative review of growth factors for induction of threedimensional in vitro chondrogenesis in human mesenchymal stem cells isolated from bone marrow and adipose tissue. Tissue Eng Part B Rev. 2010; 16:435-44. [PubMed: 20196646]

44. Shapiro F, Koide S, Glimcher MJ. Cell origin and differentiation in the repair of full-thickness defects of articular-cartilage. J Bone Joint Surg Am. 1993; 75A:532-53. [PubMed: 8478382]

45. Murakami N, Saito N, Horiuchi H, Okada T, Nozaki K, Takaoka K. Repair of segmental defects in rabbit humeri with titanium fiber mesh cylinders containing recombinant human bone morphogenetic protein-2 (rhbmp-2) and a synthetic polymer. J Biomed Mater Res. 2002; 62:16974. [PubMed: 12209936]

46. Chen FM, Chen R, Wang XJ, Sun HH, Wu ZF. In vitro cellular responses to scaffolds containing two microencapulated growth factors. Biomaterials. 2009; 30:5215-24. [PubMed: 19560814]

47. Lan J, Wang ZF, Wang YN, Wang JW, Cheng XR. The effect of combination of recombinant human bone morphogenetic and protein-2 and basic fibroblast growth factor or insulin-like growth factor-i on dental implant osseointegration by confocal laser scanning microscopy. J Periodontol. 2006; 77:357-63. [PubMed: 16512749]

48. Wang XQ, Wenk E, Zhang XH, Meinel L, Vunjak-Novakovic G, Kaplan DL. Growth factor gradients via microsphere delivery in biopolymer scaffolds for osteochondral tissue engineering. $\mathrm{J}$ Control Release. 2009; 134:81-90. [PubMed: 19071168] 
IGF-1
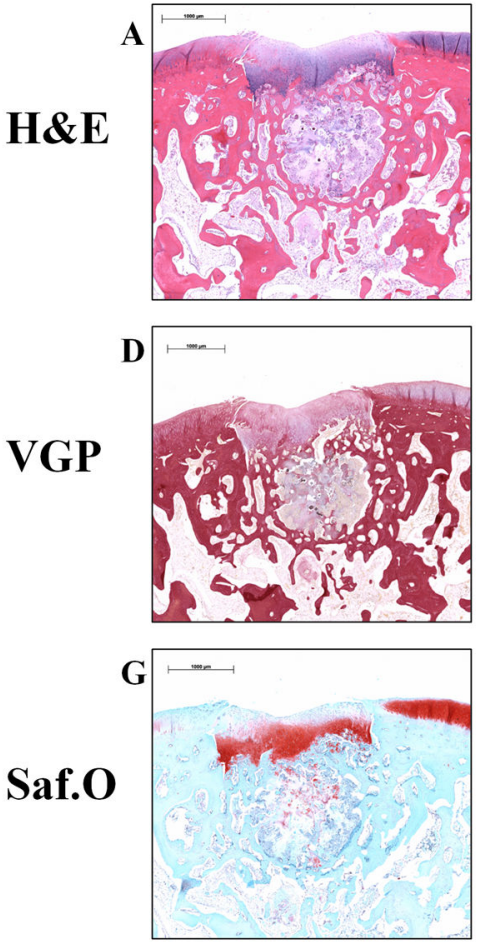

BMP-2
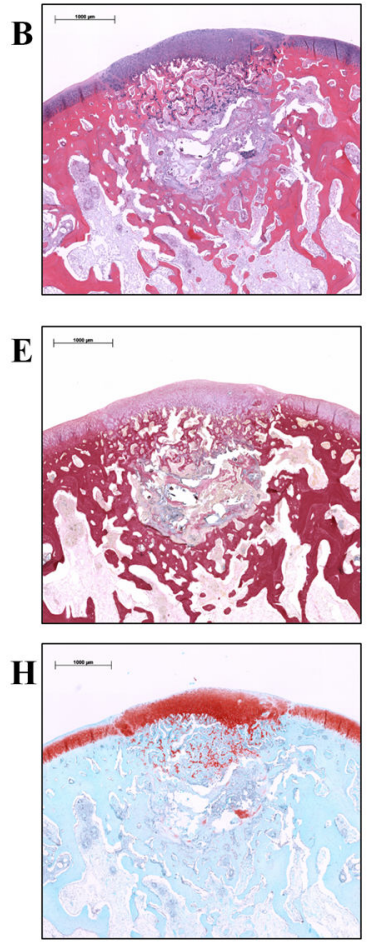

Both
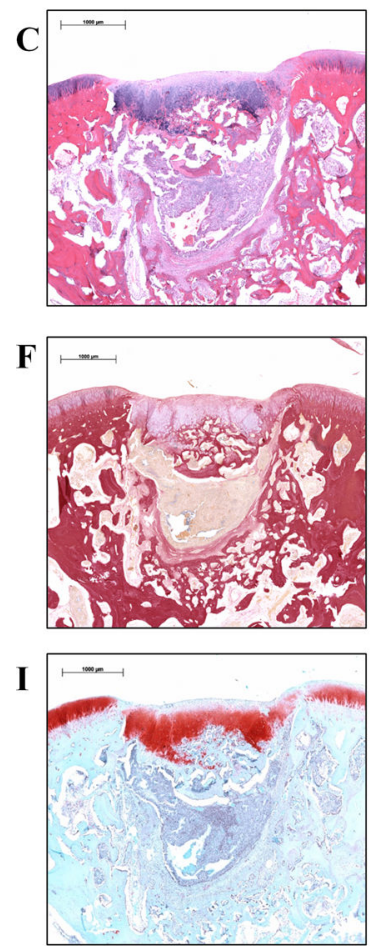

Fig. 1.

Representative histological sections of osteochondral tissue repair after 6 weeks of implantation for all three groups. Sections were stained with hematoxylin and eosin (H\&E) (A-C), van Gieson's Picrofuchsin (VGP) (D-F), or Safranin O/Fast Green (Saf.O) (G-I). All 6 week samples had incomplete degradation of the implant, evidenced by a light gray staining of the hydrogel surrounded by a yellow fibrous capsule in the VGP stains. The neocartilage formed was generally thicker than the surrounding cartilage with intense Saf.O staining and was present in the subchondral region. (scale bar: $1000 \mu \mathrm{m}$ ) 

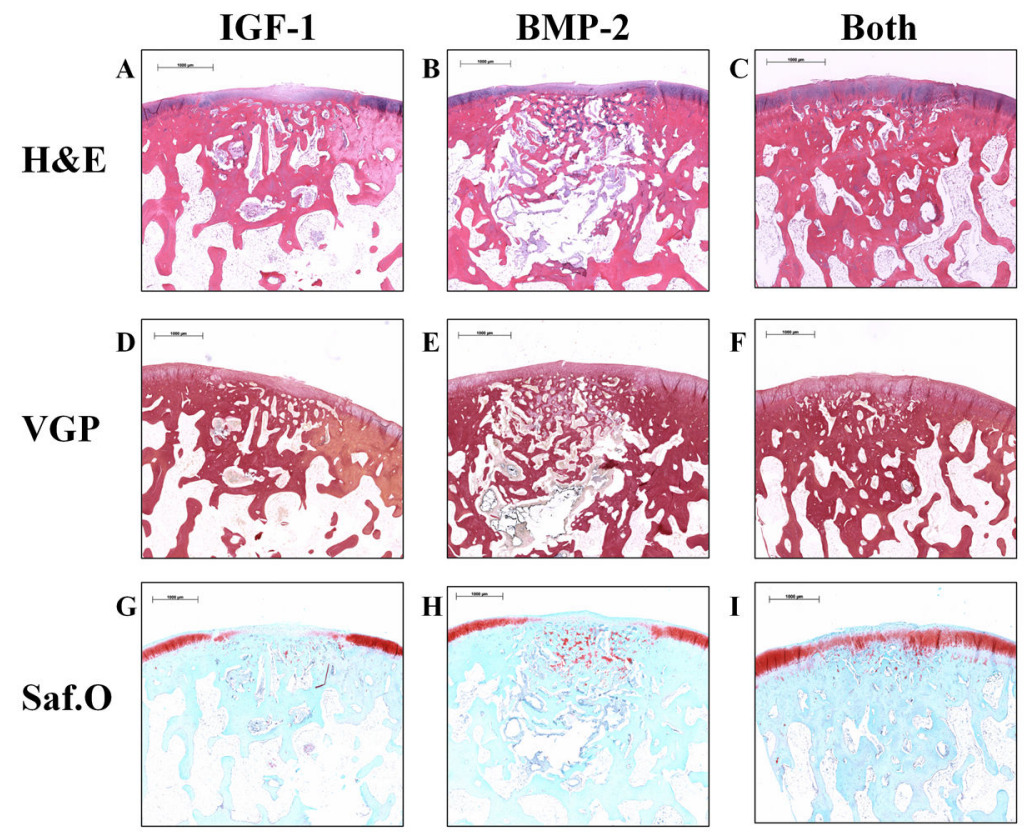

Fig. 2.

Representative histological sections of osteochondral tissue repair after 12 weeks of implantation for all three groups. Sections were stained with hematoxylin and eosin (H\&E) (A-C), van Gieson's Picrofuchsin (VGP) (D-F), or Safranin O/Fast Green (Saf.O) (G-I). 12 week samples generally had greater degradation than 6 week samples and a smaller fibrous capsule. Group IGF-1 had the most sections with fibrous tissue (D,G), group BMP-2 had the most sections with fibrocartilage $(\mathrm{E}, \mathrm{H})$, and group Both had the most sections with articular

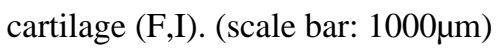



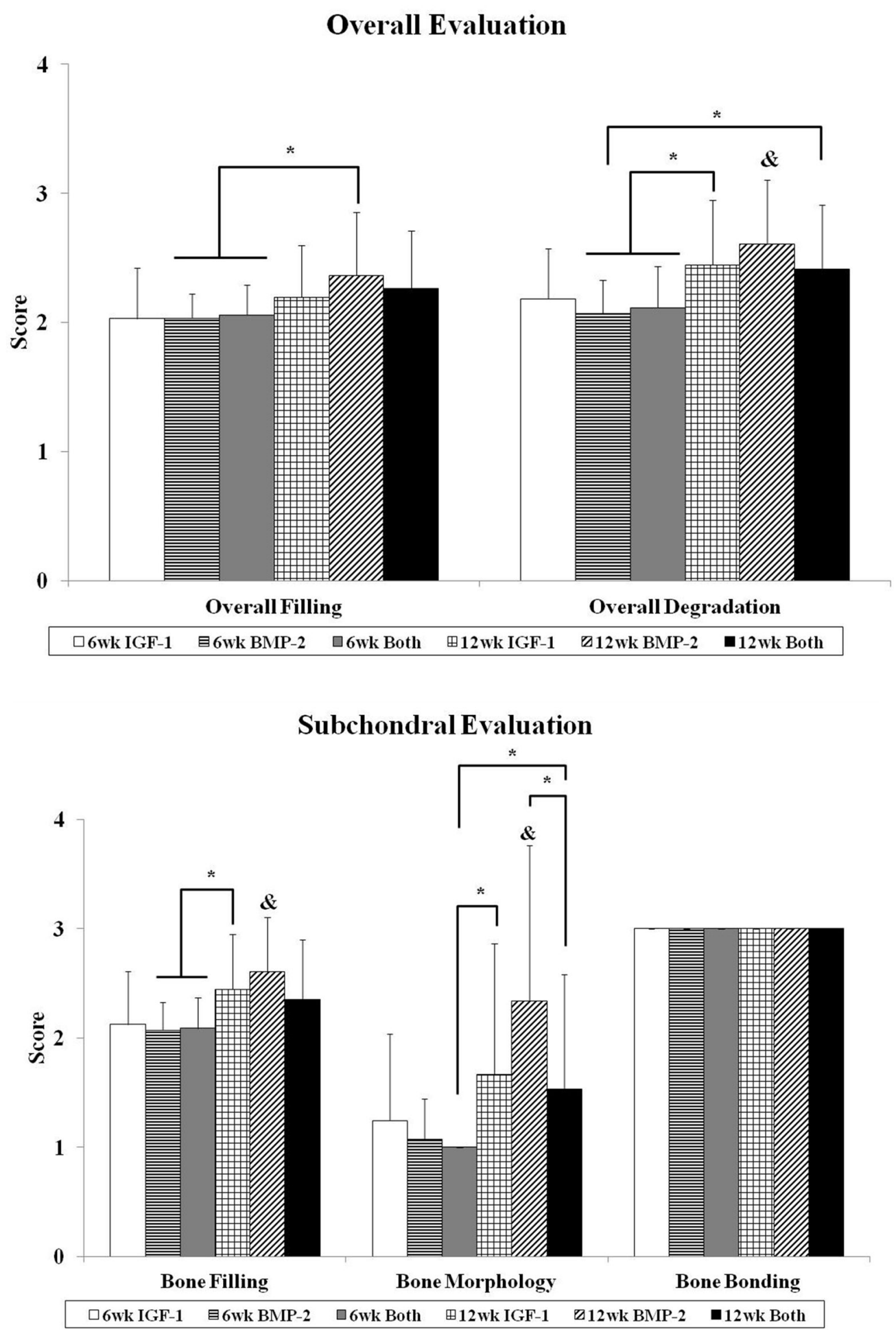

Biomaterials. Author manuscript; available in PMC 2015 October 01. 


\section{Cartilage Evaluation}

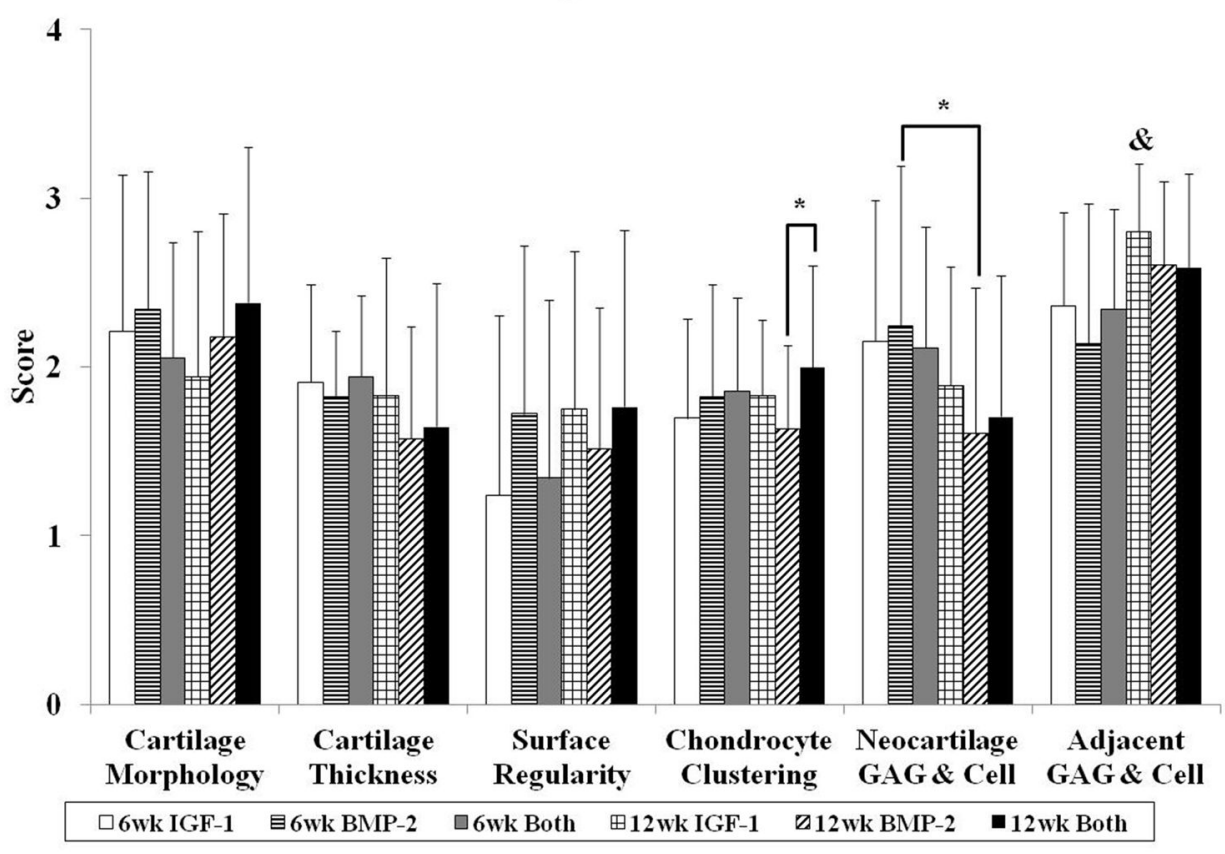

Fig. 3.

Histological scores for the overall defect evaluation (A), subchondral bone evaluation (B), and articulating cartilage evaluation (C). Data are shown as average scores with error bars representing standard deviation. $(*)$ indicates a significant difference between groups regardless of time point and $(\&)$ indicates a significant difference between a 12 week group and all 6 week groups $(\mathrm{p}<0.05)$. 
Bone Morphology

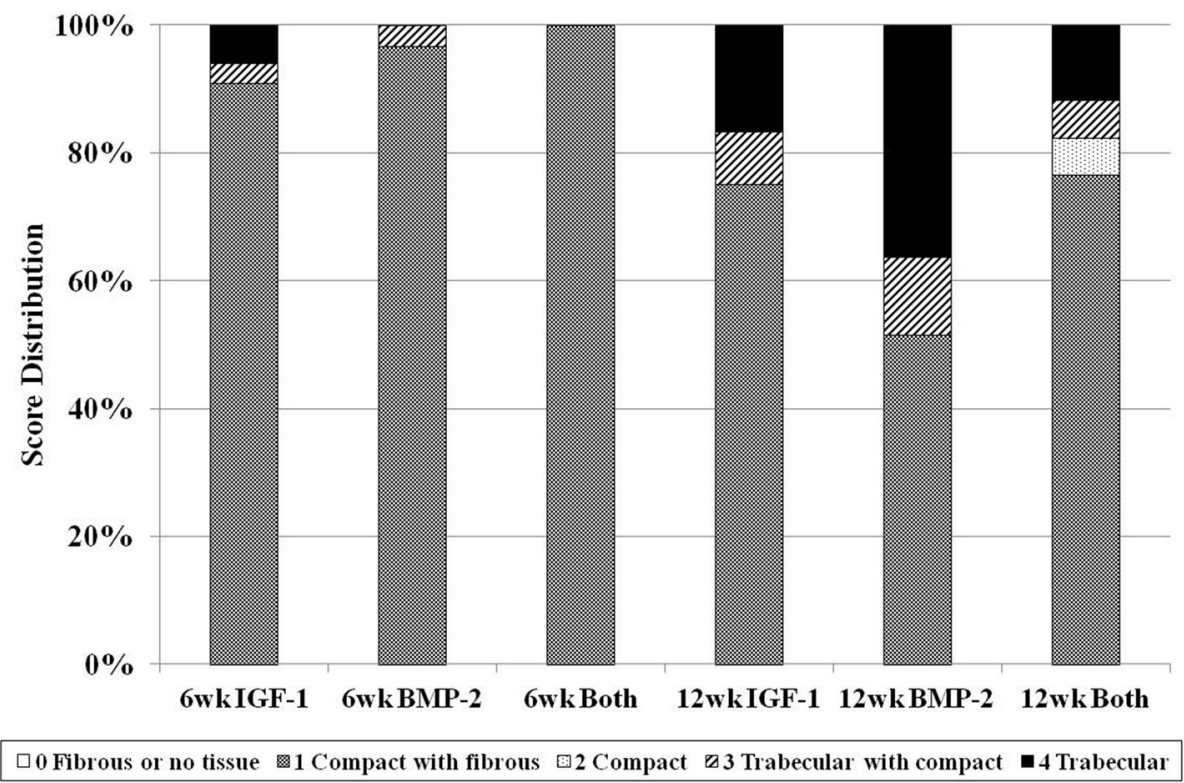

\section{Cartilage Morphology}

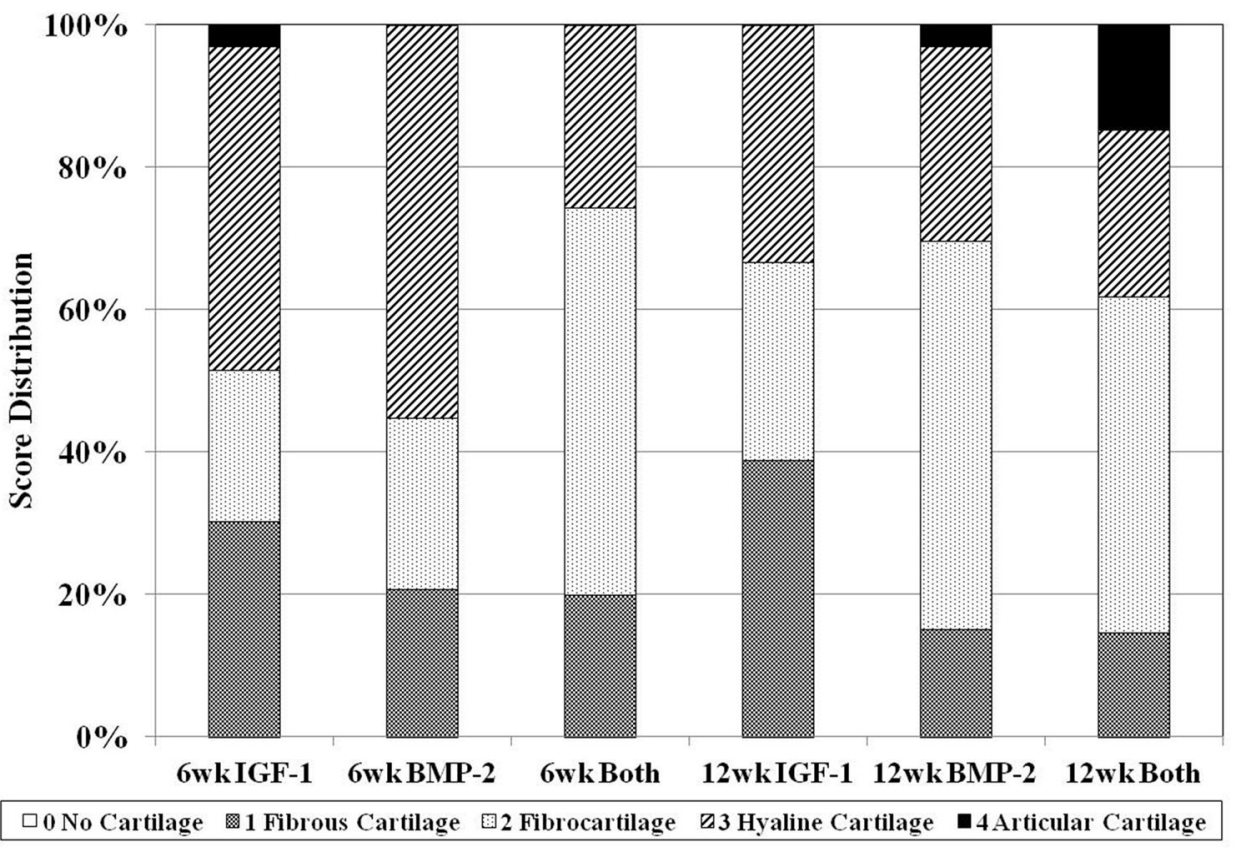




\section{Cartilage Thickness}

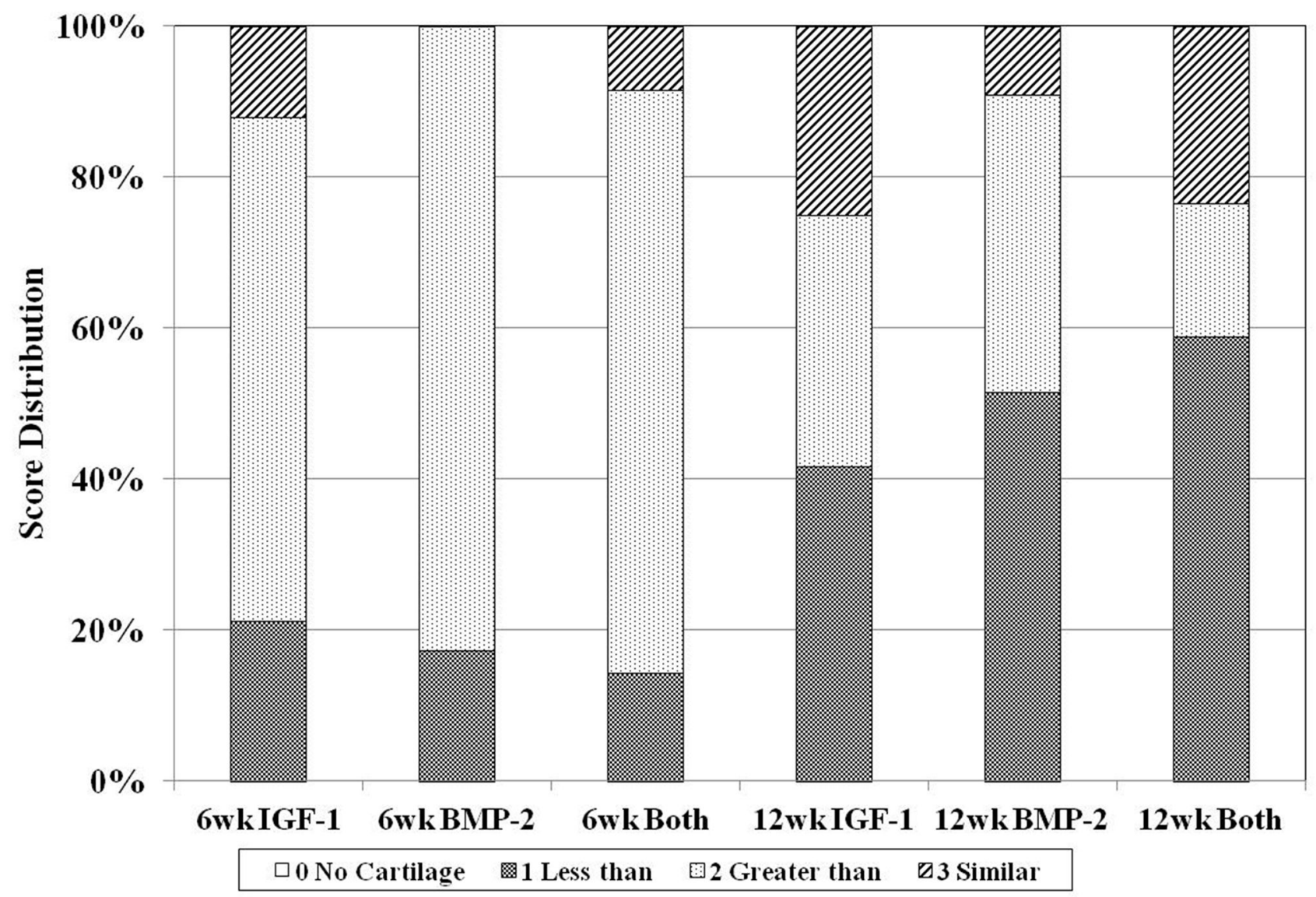

Fig. 4.

Distribution of histological scores for subchondral bone morphology (A), cartilage morphology (B), and cartilage thickness (C) for the three groups investigated at both 6 and 12 weeks. 

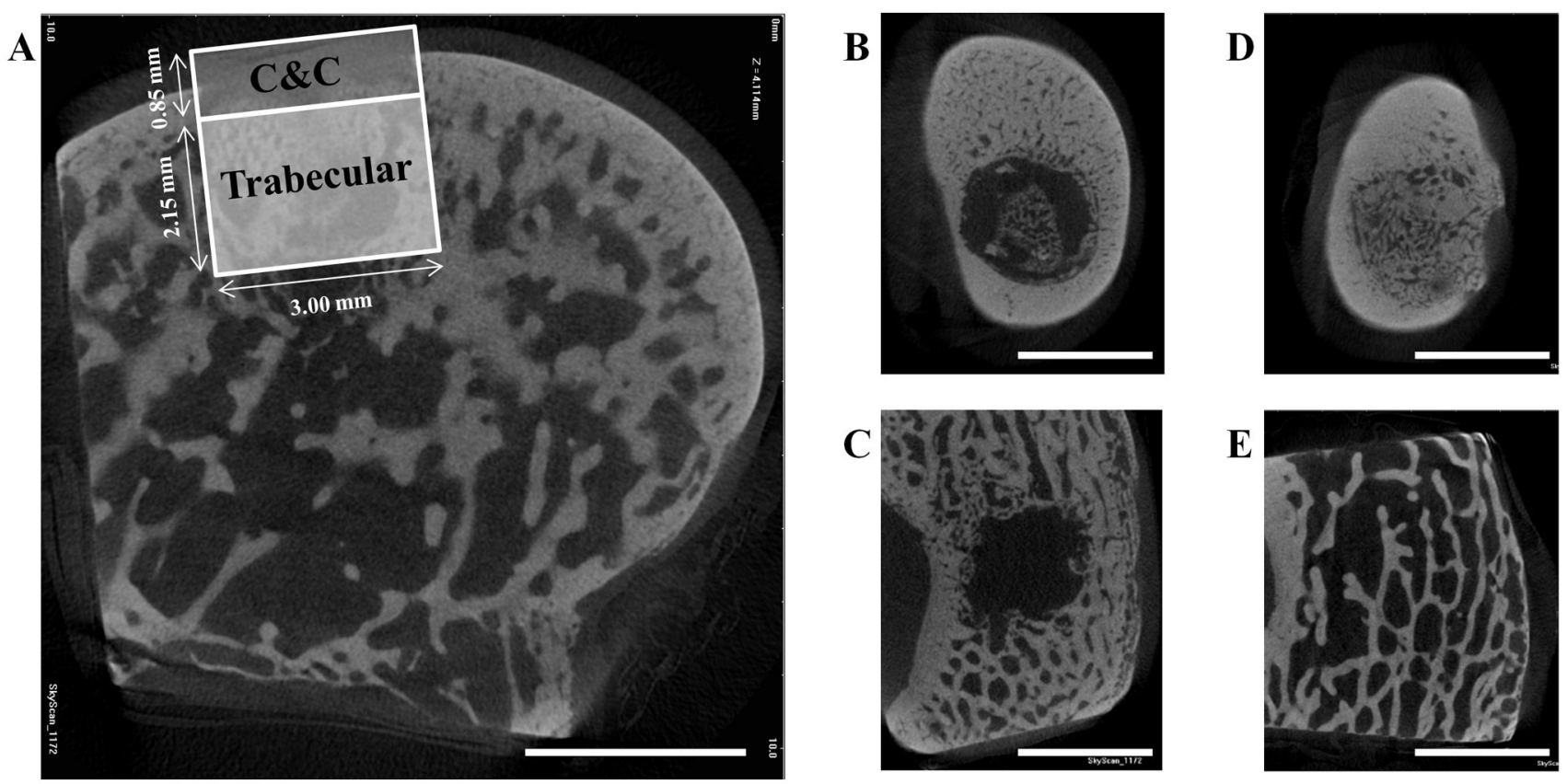

Fig. 5.

Micro-CT images showing a transverse section of the osteochondral defect in a medial femoral condyle (A), sections of a 6 week Both sample in the cortical (B) and trabecular (C) region, and sections of a 12 week BMP-2 sample in the cortical (D) and trabecular (E) region. (A) also demonstrates the two volumes of interest used, cartilage and cortical (C\&C) and trabecular, when analyzing the subchondral bone. (scale bar: $3 \mathrm{~mm}$ ) 

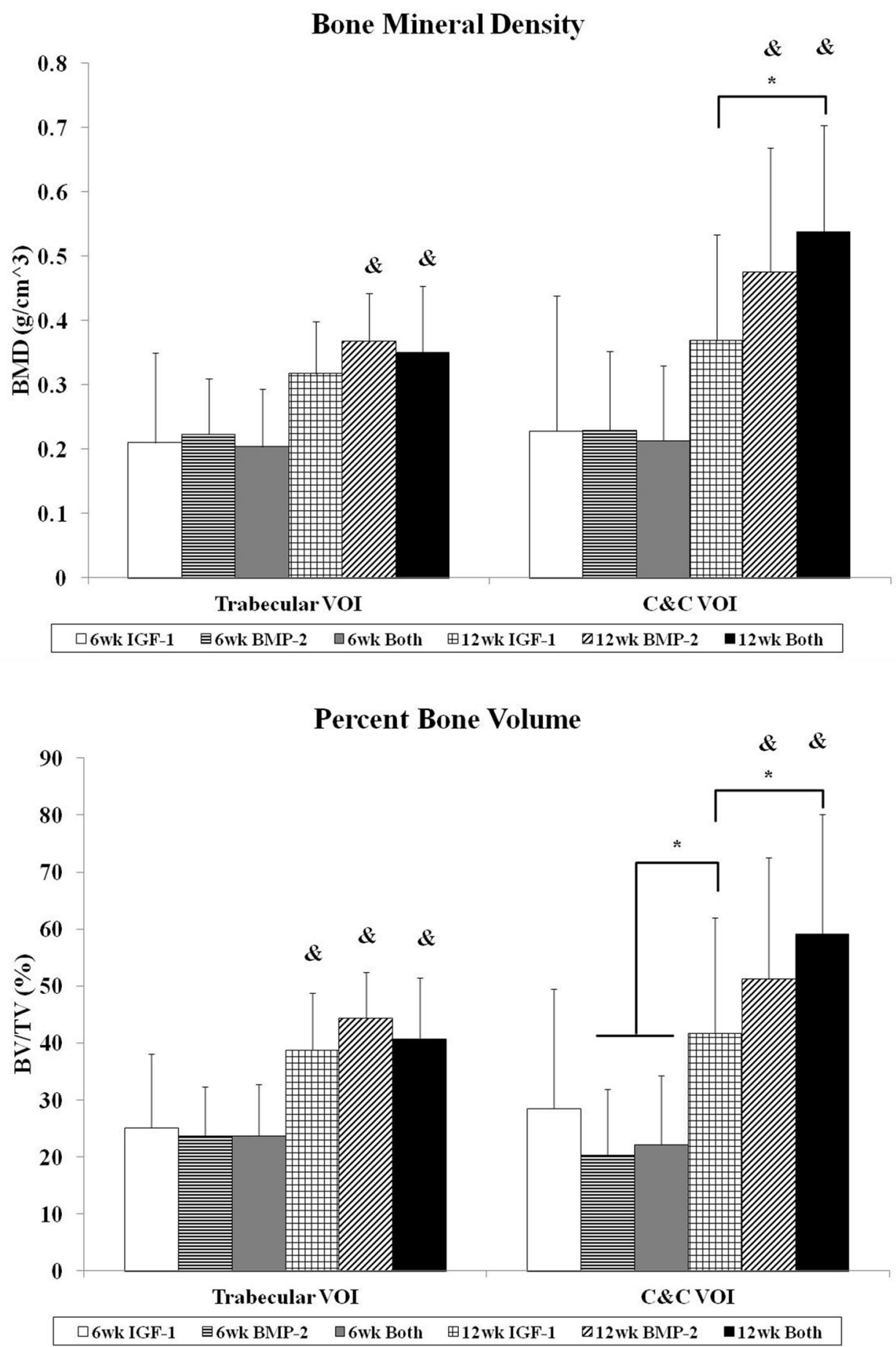

Biomaterials. Author manuscript; available in PMC 2015 October 01. 

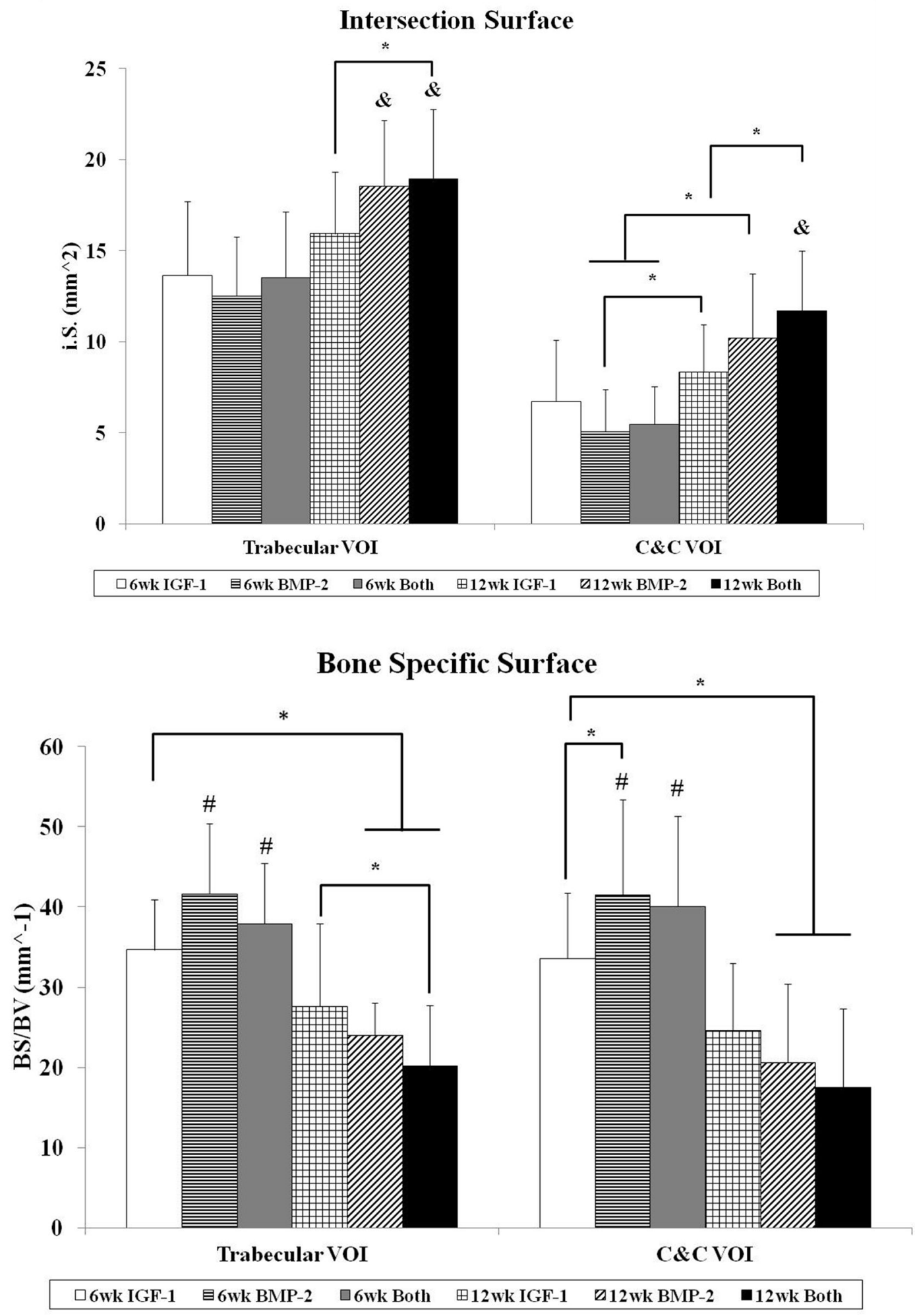

Biomaterials. Author manuscript; available in PMC 2015 October 01. 


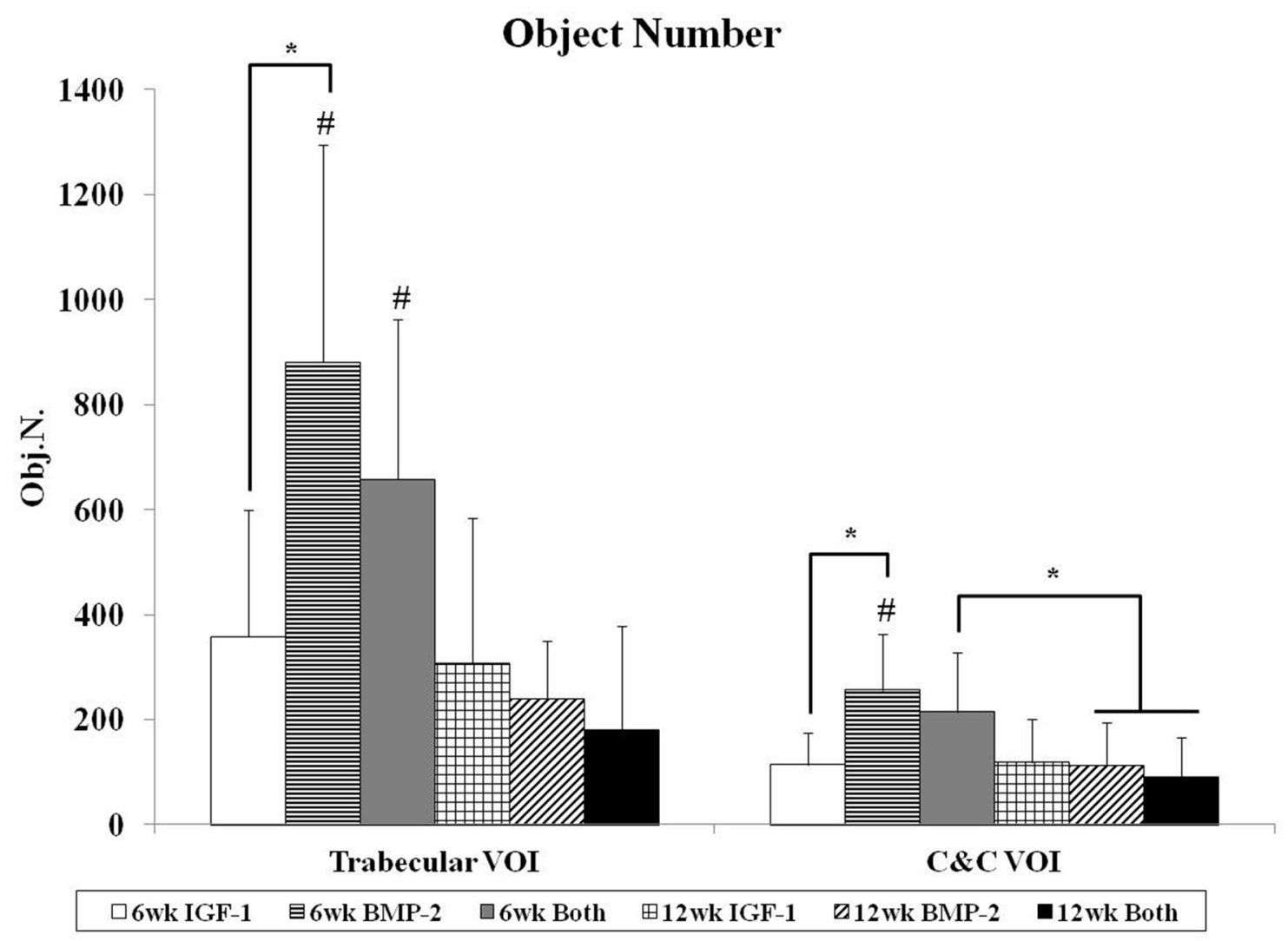

Fig. 6.

Morphological parameter analysis for both trabecular and cartilage and cortical (C\&C) volumes of interest (VOI). Bone mineral density (A), percent bone volume (B), intersection surface (C), bone specific surface (D), and object number (E) were measured for all groups at both time points. Data are shown as average scores with error bars representing standard deviation. $(*)$ indicates a significant difference between groups regardless of time point, $(\&)$ indicates a significant difference between a 12 week group and all 6 week groups, and (\#) indicates a significant difference between a 6 week group and all 12 week groups $(\mathrm{p}<0.05)$. 


\section{Table 1}

Experimental groups investigated in this study. Growth factors were loaded into gelatin microparticles prior to encapsulation within bilayered OPF hydrogels.

\begin{tabular}{cccc}
\hline Experimental Groups & IGF-1 & BMP-2 & Both \\
\hline $\begin{array}{c}\text { Chondral Layer (Top 1mm) } \\
\text { Subchondral Layer (Bottom 2mm) }\end{array}$ & IGF-1 & Blank & IGF-1 \\
\hline 6 Week Repetitions & 12 & 10 & 12 \\
12 Week Repetitions & 12 & 11 & 12 \\
\hline
\end{tabular}


Table 2

Histological scoring system for the evaluation of (a) overall tissue, (b) subchondral bone, and (c) cartilage in rabbit osteochondral defects.

Score

\begin{tabular}{lr}
\hline & S \\
\hline (a) Overall defect evaluation (throughout the entire defect depth) & \\
1. Percent filling with newly formed tissue & 3 \\
$100 \%$ & 2 \\
$>50 \%$ & 1 \\
$<50 \%$ & 0 \\
$0 \%$ & \\
2. Percent degradation of the implant & 3 \\
$100 \%$ & 2 \\
$>50 \%$ & 1 \\
$<50 \%$ & 0 \\
$0 \%$ &
\end{tabular}

(b) Subchondral bone evaluation (within the bottom $2 \mathrm{~mm}$ of defect)

3. Percent filling with newly formed tissue

$100 \%$

$>50 \%$

$<50 \%$

1

$0 \%$

4. Subchondral bone morphology

Normal, trabecular bone

Trabecular bone, with some compact bone 3

Compact bone $\quad 2$

Compact bone and fibrous tissue 1

Only fibrous tissue or no tissue 0

5. Extent of new tissue bonding with adjacent bone

Complete on both edges 3

Complete on one edge 2

Partial on both edges 1

Without continuity on either edge 0

(c) Cartilage evaluation (within the upper $1 \mathrm{~mm}$ of defect)

6. Morphology of newly formed surface tissue

Exclusively articular cartilage $\quad 4$

Mainly hyaline cartilage 3

Fibrocartilage (spherical morphology observed with $275 \%$ of cells) 2

Only fibrous cartilage (spherical morphology observed with $<75 \%$ of cells) $\quad 1$

No tissue 0

7. Thickness of newly formed cartilage

Similar to the surrounding cartilage 3

Greater than the surrounding cartilage $\quad 2$ 


\begin{tabular}{ll}
\hline & Score \\
\hline Less than the surrounding cartilage & 1 \\
No cartilage & 0 \\
8. Joint surface regularity & 3 \\
Smooth, intact surface & 2 \\
Surface fissures (<25\% of new surface thickness) & 1 \\
Deep fissures ( $25 \%$ of new surface thickness) & 0 \\
Complete disruption of the new surface & \\
9. Chondrocyte clustering & 3 \\
None at all & 2 \\
<25\% chondrocytes & 1 \\
25-100\% chondrocytes & 0 \\
No chondrocytes present (no cartilage) & \\
10. Chondrocyte and GAG content of new cartilage & 3 \\
Normal cellularity with normal Safranin O staining & 2 \\
Normal cellularity with moderate Safranin O staining & 1 \\
Clearly less cells with poor Safranin O staining & 0 \\
Few cells with no or little Safranin O staining or no cartilage & 2 \\
11. Chondrocyte and GAG content of adjacent cartilage & 3 \\
Normal cellularity with normal Safranin O staining & \\
Normal cellularity with moderate Safranin O staining & \\
Clearly less cells with poor Safranin O staining & 2 \\
Few cells with no or little Safranin O staining or no cartilage & \\
\hline
\end{tabular}




\title{
Table 3
}

Explanation of 3D morphometric parameters analyzed within two volumes of interest (VOI): the cartilage and cortical region and the trabecular region.

\author{
Bone mineral density $\left(\mathrm{g} / \mathrm{cm}^{3}\right)$ BMD Volumetric density of bone within a mixed bone-soft tissue VOI \\ Percent bone volume (\%) BV/TV Proportion of VOI occupied by binarised solid objects (e.g., bone) \\ Intersection surface $\left(\mathbf{m m}^{2}\right)$ i.S. Surface of VOI intersected by bone, useful for evaluating bone growth at a defined boundary \\ Bone specific surface $\left(\mathbf{m m}^{-\mathbf{1}}\right) \mathbf{B S} / \mathbf{B V}$ Ratio of bone surface to bone volume within the VOI, useful for characterizing the relative \\ complexity of structures \\ Object number Obj.N. Total number of discreet binarised objects within the VOI, where a discreet object is a connected \\ cluster of solid (white) voxels fully surrounded by space (black) voxels
}




\section{Table 4}

Main effects analysis of timepoint (week 6 vs. week 12) for histological scoring and micro-CT morphometric parameters $(\mathrm{p}<0.05)$.

\begin{tabular}{cccc}
\hline Histological scoring parameters & Micro-CT morphometric parameters \\
\hline Overall Filling & $12 w \mathrm{w}>6 \mathrm{wk}$ & Bone Mineral Density & $12 \mathrm{wk}>6 \mathrm{wk}$ \\
Overall Degradation & $12 \mathrm{wk}>6 \mathrm{wk}$ & Percent Bone Volume & $12 \mathrm{wk}>6 \mathrm{wk}$ \\
Bone Filling & $12 \mathrm{wk}>6 \mathrm{wk}$ & Intersection Surface & $12 \mathrm{wk}>6 \mathrm{wk}$ \\
Bone Morphology & $12 \mathrm{wk}>6 \mathrm{wk}$ & Bone Specific Surface & $6 \mathrm{wk}>12 \mathrm{wk}$ \\
Bone Bonding & -- & Object Number & $6 w \mathrm{wk} 12 \mathrm{wk}$ \\
Cartilage Morphology & -- & & \\
Cartilage Thickness & $6 w \mathrm{wk}>12 \mathrm{wk}$ & & \\
Surface Regularity & -- & & \\
Chondrocyte Clustering & -- & & \\
Neocartilage GAG \& Cell & $6 w \mathrm{k}>12 \mathrm{wk}$ & & \\
Adjacent GAG \& Cell & $12 w \mathrm{wk}>6 \mathrm{wk}$ & & \\
\hline
\end{tabular}

